\title{
Children are not just little adults: recent advances in understanding of diffuse intrinsic pontine glioma biology
}

\author{
Kristin M. Schroeder', Christine M. Hoeman' ${ }^{1}$ and Oren J. Becher ${ }^{1,2}$
}

Diffuse intrinsic pontine glioma (DIPG) is a high-grade glioma that originates in the pons and is seen exclusively in children. Despite numerous efforts to improve treatment, DIPG remains incurable with $90 \%$ of children dying within 2 y of diagnosis, making it one of the leading causes of death in children with brain tumors. With the advent of new genomic tools, the genetic landscape of DIPG is slowly being unraveled. The most common genetic alterations include a K27M mutation in $\mathrm{H} 3.3$ or H3.1, which are found in up to $78 \%$ of DIPGs, whereas p53 mutations are found in up to $77 \%$. Other recently discovered alterations include amplification of components of the receptor tyrosine kinase/Ras/phosphatidylinositol 3-kinase signaling pathway, particularly platelet-derived growth factor receptor A. Recapitulating such alterations, genetically engineered DIPG preclinical models have been developed, and DIPG xenograft models have also been established. Both models have strengths and weaknesses but can help with the prioritization of novel agents for clinical trials for children with DIPG. As we move forward, it is important that we continue to study the complex and unique biology of DIPG and develop improved preclinical models to increase our understanding of DIPG pathogenesis, allowing translation into successful therapies in the not too distant future.

A n estimated 4,000 new malignant and nonmalignant brain tumors are diagnosed annually in children in the United States $(1,2)$. Fifteen percent of malignant central nervous system tumors in children younger than $20 \mathrm{y}$ of age arise in the brainstem, with the majority being diffuse intrinsic pontine glioma (DIPG) subtype (1,3). DIPG is a high-grade glioma (HGG) that originates in the pons and is seen almost exclusively in children with a median age of diagnosis of 6-7 y (4-6). Despite numerous efforts to improve treatment, prognosis remains poor with more than $90 \%$ of children dying within $2 \mathrm{y}$ of diagnosis, making it one of the major causes of brain-related death in children $(4,7)$.

A key to improving these outcomes is to gain a better understanding of DIPG tumor biology. Historically, pediatric HGGs were thought to resemble adult HGGs, and children were treated with the same drugs that were being evaluated in adults. However, new biologic, molecular, and genetic data have clearly demonstrated that pediatric DIPG have distinct genetic alterations as compared with adult HGGs and even with pediatric supratentorial HGGs. This strongly suggests that effective therapies for DIPG may be distinct from effective therapies for HGGs. This review will present an overview of DIPG presentation and current therapy and discuss how new unique genetic findings are shaping future research.

\section{PRESENTATION AND DIAGNOSIS}

Patients with DIPG typically present with less than 3 mo of preceding neurological symptoms that can vary based on lesion location. Over $50 \%$ present with the "classic" triad of cranial nerve deficits (diplopia and facial asymmetry), long tract signs (hyperrefflexia, upward Babinski, and decreased strength), and cerebellar signs (ataxia, dysmetria, and dysarthria) $(8,9)$. Abducens palsy (cranial nerve VI) is almost always the first clinical sign, and its presence is a highly sensitive predictor of DIPG (9). Signs of increased intracranial pressure are not typically seen, but in $<10 \%$ of patients the tumor may extend posteriorly and cause obstructive hydrocephalus. DIPG tumors rarely metastasize to distant sites but can expand along known fiber tracts into the cerebellum/ thalamus (10).

DIPG diagnosis is based on clinical history-including physical symptoms and time to presentation-combined with radiologic magnetic resonance imaging findings. Typical DIPG tumors are hypointense with indistinct margins on magnetic resonance imaging T1-weighted images, whereas they are hyperintense on T2-weighted/fluid-attenuated inversion recovery images (Figure 1) $(4,9)$. Gadolinium enhancement is often absent or minimal, which distinguishes DIPG from pilocytic astrocytomas or other central nervous system tumors (11). Historically, classic findings on physical exam and imaging were sufficient for DIPG diagnosis, and biopsy confirmation was thought to be an unnecessary risk because it did not change disease management (12). The lack of clear biopsy benefit combined with improved diagnostic imaging capabilities led to magnetic resonance imaging scans becoming the diagnostic standard of care for DIPGs in the United States.

In France, biopsies have been routinely performed since 2003 for both atypical and typical DIPG evaluation and have been associated with minimal morbidity and high diagnostic 
a

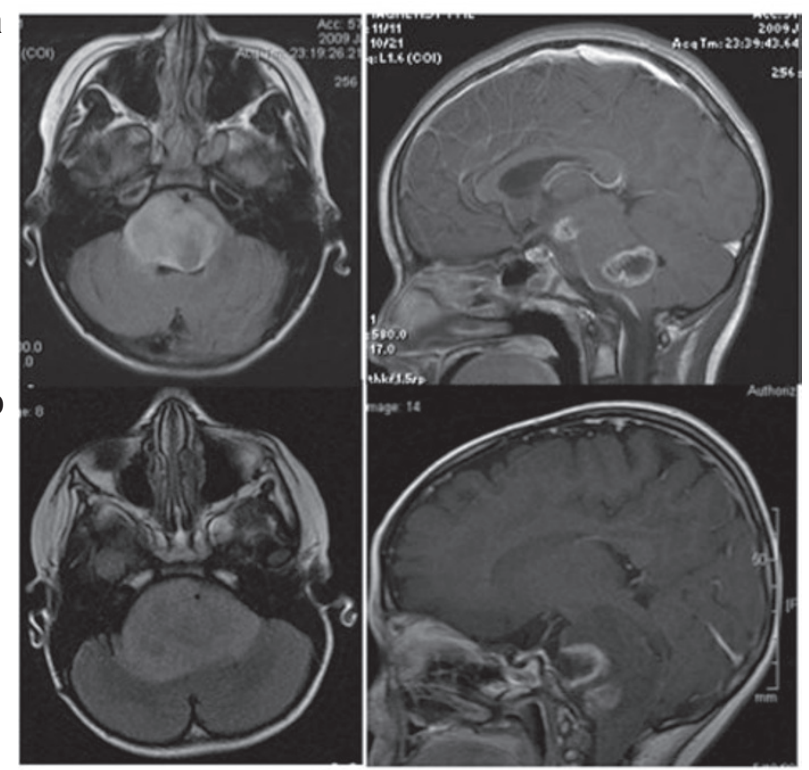

Figure 1. Typical magnetic resonance imaging images of diffuse intrinsic pontine glioma (DIPG). Axial fluid-attenuated inversion recovery (left) and sagittal T1 with contrast (right) of a (a) more dorsally located DIPG and

(b) more ventrally located DIPG. Figure reproduced with permission from Oxford University Press.

yield (13). This surgical success has also led to an increase in available DIPG tumor samples, providing much of the new biologic, molecular, and genetic data that are currently advancing our understanding of DIPG biology. Some of the identified biologic markers have been shown to correlate with progression-free survival and may be useful to stratify patients in future clinical trials (14). With the potential for new therapeutic and diagnostic options combined with the relative low morbidity, the benefit of biopsies is being reconsidered and may eventually be included as part of the routine diagnostic evaluation for DIPG in the United States (15).

\section{TREATMENT}

\section{Radiotherapy}

The standard treatment for patients with DIPG remains conventional radiotherapy with a treatment dose of 54-60 Gy, fractionated over a 6-wk period (7). This treatment has been shown to transiently improve neurologic function or temporarily stabilize disease in $70 \%$ of patients, but the effect on overall survival is minimal, with mean progression-free survival of $5.8 \mathrm{mo}$ compared with 5 mo without radiotherapy (4). In an effort to improve quality of life for children with DIPG, hypofractionated radiotherapy was evaluated, shortening the time children spent in the hospital. Limiting radiation to 3 Gy daily for $3 \mathrm{wk}$ reduced toxicity without negatively impacting overall survival (16).

\section{Chemotherapy/Targeted Agents}

With the known benefit of radiation, numerous studies have looked at combining traditional cytotoxic chemotherapeutic agents or targeted therapeutics before, during, and after radiotherapy. In 2006, Hargrave et al. (7) reviewed 29 clinical studies completed in 1984-2005, and in 2012, Jansen et al. (17) reviewed an additional 26 studies completed in 2005-2011, and none of the studies demonstrated improvement in the response rate, event-free survival, or overall survival in children with DIPG. It is worth noting that numerous combinations of cytotoxic agents have been evaluated in this disease, but very few combinations of targeted agents have been tested.

\section{Drug Delivery Strategies}

The lack of efficacy of systemic chemotherapy or targeted agents may be due to the inherent resistance of DIPG cells to such treatments, or alternatively due to inadequate delivery of these agents into the tumor. In support of the latter explanation, it is well-known that DIPGs have a relatively intact bloodbrain barrier as evident by the minimal contrast enhancement with magnetic resonance imaging. This impermeability is due to tight junctions between the endothelial cells combined with limited transcellular transport and expression of adenosine triphosphate-binding cassette transporters such as multidrug resistance (MDR) and adenosine triphosphate-binding cassette subfamily $\mathrm{G}$ member 2 on the endothelial cells. To bypass the blood-brain barrier in DIPG, several approaches have been evaluated thus far, with limited success, including hyperosmotic solutions (18), bradykinin analogs (19), and an MDR inhibitor (20). No survival benefit was seen, and additionally, the MDR inhibitor increased chemotherapy toxicity.

Direct infusion of the therapeutic agent into the tumor bed via convection-enhanced delivery (CED) is another approach that is now gaining momentum. CED uses a stereotactically placed catheter with attached pump to provide positive pressure and maintain convective flow, bypassing the blood-brain barrier and providing direct localized drug delivery into the tumor (21). Based on moderate success in adult trials, a recent pediatric DIPG phase I trial using CED with topotecan demonstrated technical feasibility, but with increased chemotherapy volume and flow, there was significant morbidity (22). The optimal infusion rate, infusion time, and agent continue to be debated, but CED remains a promising approach for the treatment of DIPG.

\section{GENETIC ALTERATIONS}

With the advent of genomic tools, the genetic landscape of DIPG is slowly being unraveled. Several molecular studies have shown that DIPG have distinct alterations when compared with adult HGG and non-DIPG pediatric HGG (Table 1). For example, Paugh et al. (23) completed the largest DIPG autopsy cohort study with $n=43$ and found that gain of $1 \mathrm{q}$ is more common in DIPG compared with that in adult HGG, and alternately, epidermal growth factor receptor amplification, which is one of the most frequently amplified genes in adult HGG, was rarely seen in DIPG autopsy samples. Compared with nonbrainstem HGG, DIPG have an increased frequency of $17 \mathrm{p}$ loss and an absence of CDKN2A or CDKN2B deletion $(23,24)$. In the first comprehensive genomic analysis of diagnostic biopsy DIPG samples, Puget et al. (25) demonstrated that DIPG have distinct gene expression profiles compared with pediatric supratentorial HGG, with increased expression of homeobox and HLH 


\section{Advances in the understanding of DIPG $\quad$ Review}

Table 1. Distinct molecular genetics of DIPG as compared with pediatric high-grade glioma (non-DIPG) and adult high-grade gliomas

\begin{tabular}{|c|c|c|c|c|}
\hline & DIPG (\%) & $\begin{array}{c}\text { Pediatric high-grade } \\
\text { glioma (non-DIPG) (\%) }\end{array}$ & $\begin{array}{l}\text { Adult high-grade } \\
\text { glioma (\%) }\end{array}$ & References \\
\hline ATRX mutation & 9 & 31 & 14 & $(28,36)$ \\
\hline EGFR amplification & $0-18$ & $0-19$ & $40-55$ & $(37-44)$ \\
\hline H3F3A mutation-K27M & $60-71$ & 19 & $<3$ & $(28,30,36)$ \\
\hline IDH1/2 mutations & 0 & $10-16.3$ & 42 & $(28,37,45,46)$ \\
\hline TP53 mutation & $40-77$ & $21-54$ & $33-43$ & $(27,28,38,47,48)$ \\
\hline SETD2 mutation & NA & 15 & 8 & (49) \\
\hline PDGFR-A amplification & $13-36$ & $4-10$ & 11 & $(23-25,29,37,39)$ \\
\hline Gain 8q & 28 & 5 & 5 & (23) \\
\hline Gain 9q & 28 & 10 & 8 & (23) \\
\hline Gain $7 p / 7 q$ & $14 / 9$ & $13 / 15$ & $70 / 74$ & $(23,37)$ \\
\hline Loss $16 q$ & 49 & $18-26$ & 7 & $(23,37,50)$ \\
\hline Loss $17 p$ & $31-64$ & $4-25$ & 9 & $(23,24,39,50)$ \\
\hline Loss $20 p$ & 26 & 3 & 1 & (23) \\
\hline Loss $21 \mathrm{q}$ & 2 & 21 & 3 & (23) \\
\hline Loss $3 q$ & 0 & 21 & 4 & (23) \\
\hline Loss $4 q$ & 7 & $21-54$ & 2 & $(23,24,37,43)$ \\
\hline
\end{tabular}

ATRX, a-thalassemia/mental retardation syndrome X-linked; CDKN2A/B, cyclin dependent kinase inhibitor 2A/2B; DIPG, diffuse intrinsic pontine glioma; EGFR, epidermal growth factor receptor; NA, not available; PDGFR-A, platelet-derived growth factor receptor A; PTEN, phosphatase and tensin homolog.

genes in the DIPG samples. In contrast to adult HGGs, isocitrate dehydrogenase $1 / 2$ mutations were not observed in this cohort (26). In addition, two distinct DIPG subgroups were identified: oligodendroglial, associated with platelet-derived growth factor receptor A amplification, and a second mesenchymal and proangiogenic phenotype (25).

Other known mutations in DIPG include p53 mutations in $75 \%$ of DIPG samples, PIK3CA mutations in $15 \%$ of DIPG samples, and platelet-derived growth factor receptor A mutations in $10 \%$ of DIPG samples $(25,27,28)$. In addition to mutations, up to $50 \%$ of DIPGs harbor gains in components of the receptor tyrosine kinase/Ras/phosphatidylinositol 3-kinase/v-akt murine thymoma viral oncogene homolog 1 (AKT) signaling network with gains in platelet-derived growth factor receptor $\mathrm{A}$ as the most commonly amplified component. Other components include $c-M E T$ (also known as hepatocyte growth factor receptor), insulin-like growth factor receptor, insulin-like growth factor 2, hepatocyte growth factor, $A K T 1$, Kirsten rat sarcoma viral oncogene homolog (KRAS), PIK3CA, PIKC2G, epidermal growth factor receptor, erythroblastic leukemia viral oncogene homolog-4 (ERBB4), and AKT3. Cell-cycle control genes are also gained in up to $30 \%$ of DIPGs such as CDK4, CDK6, and D-type cyclins $(23,24,29)$. Finally, in addition to the above-mentioned loss of $17 \mathrm{p}$, there are other broad chromosomal gains and losses that are particularly common in DIPGs compared with nonbrainstem HGG, including gains of chromosomes 2q, 8q, and 9q and losses of 16q and 20p $(23,29)$.

Using whole-genome sequencing, Wu et al. (30) examined 7 DIPGs combined with targeted sequencing from 43 additional DIPG and nonbrainstem HGG samples. Over 75\% of DIPG samples were found to have mutations in either H3F3A or HIST1H3B, which encode histone variant $\mathrm{H} 3.3$ or $\mathrm{H} 3.1$, respectively, resulting in a Lys27Met amino acid substitution. This residue is in the highly conserved $\mathrm{N}$-terminal of the histone $\mathrm{H} 3$ protein, and mutations can effect the epigenetic regulation of gene expression, including nucleosome structure and interactions between the histone complex and transcription modifiers. Previous studies have shown either methylation or acetylation of histone H3 Lys27 can control gene activation $(31,32)$. Most recently, Lewis et al. (33) demonstrated that the K27M histone mutation is a gain of function mutation that inhibits the activity of the polycomb repressive complex 2 . The exact mechanism by which inhibition of polycomb repressive complex 2 contributes to DIPG pathogenesis is currently the subject of intense investigation.

\section{DIPG MOUSE MODELS}

Prior to 2010, researchers used adult glioma cell lines implanted into the brainstem of immunodeficient mice as DIPG 
preclinical tools, with the assumption that the genetic alterations of adult gliomas are similar to the genetic alterations of DIPG. Since 2010, two complementary approaches have been used to develop improved DIPG mouse models: DIPG xenograft models and genetically engineered DIPG mouse models. Monje et al. (34) have successfully cultured DIPG tumor cells from postmortem tissue and implanted them into immunodeficient mice to create the first-published DIPG xenograft model. The advantages of this approach are that this model arose from an actual DIPG human tumor with all of its complex genomic heterogeneity. A disadvantage of this model is that using immunodeficient mice removes the immune system as a component of DIPG biology. A second disadvantage is that this modeling approach assumes that the complex stroma-tumor interactions in DIPG are conserved when one uses human tumor cells with a mouse stroma.

Alternately, a genetically engineered mouse model of DIPG was developed by Becher and colleagues $(33,35)$, using the replication-competent ASLV long terminal repeat with a splice acceptor (RCAS)/tv-a system to overexpress PDGF-B in nestin-expressing cells, in conjunction with ink4a-ARF loss initially and more recently with p53 loss and H3.3K27M (Figure 2). Tumors generated using this model have histologic and immunophenotypic similarities to human DIPG samples, and this mouse model has been successfully used in preclinical screening. Advantages of the genetically engineered mouse modeling approach include the ability to generate tumors in the brainstem of immunocompetent mice and the ability to study the contribution of each genetic alteration to DIPG biology. In addition, tumors arising with this approach are infiltrating, invading the surrounding normal brain as seen in human DIPG (Figure 2d). However, this genetic mouse modeling

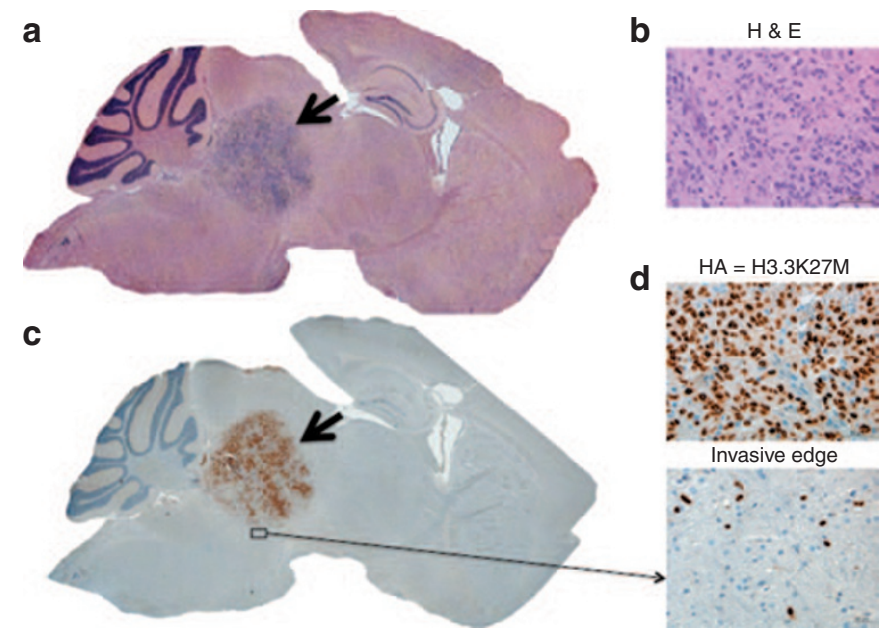

Figure 2. A genetically engineered mouse model of H3.3K27M mutant diffuse intrinsic pontine glioma (DIPG). (a) Sagittal hematoxylin and eosin (H\&E) image of DIPG-bearing mouse (PDGF-B; H3.3K27M; p53 loss). The arrow points to tumor. (b) $40 \times$ Magnification H\&E of DIPG-bearing mouse from a. Scale bar $=50 \mu \mathrm{m}$. (c) Sagittal immunohistochemistry image for hemagglutinin ( $\mathrm{HA})(\mathrm{H} 3.3 \mathrm{~K} 27 \mathrm{M}$ is $(\mathrm{HA})$ tagged) of DIPG-bearing mouse from a. The arrow points to tumor. (d) $40 \times$ Magnification immunohistochemistry images for HA from c. Top image is of the tumor bulk and the bottom image is of the invasive edge. Scale bar $=50 \mu \mathrm{m}$. approach also has limitations. First, it is likely that DIPGs that form in this model do not have the full genetic complexity of the human disease. Second, most of the tumors arise from nestin-expressing progenitors that reside in the floor of the fourth ventricle or aqueduct of the neonatal dorsal brainstem, whereas the majority of human DIPGs are thought to arise from the ventral brainstem $(34,35)$. Finally, tumors arising with this model are more focal and usually do not occupy the entire pons as the human tumors do. In summary, both models can help to prioritize the translation of novel agents into clinical trials for children with DIPG, and preclinical models of adult gliomas should no longer be used to prioritize the translation of novel agents to treat children with DIPG.

\section{FUTURE DIRECTIONS}

Previously, novel agents that were being evaluated in clinical trials for DIPG were primarily chosen based on antitumor activity in adult glioma preclinical models; this is no longer the case. Currently, genetically engineered DIPG preclinical models and DIPG xenograft models guide future clinical trials for DIPG, and ideally, agents that demonstrate efficacy in both genetic and xenograft models should be prioritized for translation into a clinical trial $(34,35)$.

With the recent explosion in knowledge regarding the genetic alterations in DIPG, it will be important to determine the prognostic implication of each. In addition, prognostic factors that have been identified retrospectively should be evaluated prospectively in future clinical trials for children with DIPG. As we move forward, it is important that we continue to study the complex and unique biology of DIPG and develop improved preclinical models to increase our understanding of DIPG pathogenesis, allowing translation into successful therapies in the not too distant future.

\section{STATEMENT OF FINANCIAL SUPPORT}

O.B. is a Damon Runyon Clinical Investigator, a St. Baldrick's Scholar, and is supported by the US Department of Defense.

Disclosure: The authors have no relevant financial or nonfinancial statements to disclose.

\section{REFERENCES}

1. Kohler BA, Ward E, McCarthy BJ, et al. Annual report to the nation on the status of cancer, 1975-2007, featuring tumors of the brain and other nervous system. J Natl Cancer Inst 2011;103:714-36.

2. National Cancer Institute. Cancer Incidence and Survival among Children and Adolescents: United States SEER Program 1975-1995, 1999. (http://seer.cancer.gov/publications/childhood/cns.pdf.)

3. Minturn JE, Fisher MJ. Gliomas in children. Curr Treat Options Neurol 2013;15:316-27.

4. Freeman CR, Farmer JP. Pediatric brain stem gliomas: a review. Int J Radiat Oncol Biol Phys 1998;40:265-71.

5. Berger MS, Edwards MS, LaMasters D, Davis RL, Wilson CB. Pediatric brain stem tumors: radiographic, pathological, and clinical correlations. Neurosurgery 1983;12:298-302.

6. Littman P, Jarrett P, Bilaniuk LT, et al. Pediatric brain stem gliomas. Cancer 1980;45:2787-92.

7. Hargrave D, Bartels U, Bouffet E. Diffuse brainstem glioma in children: critical review of clinical trials. Lancet Oncol 2006;7:241-8.

8. Albright AL, Guthkelch AN, Packer RJ, Price RA, Rourke LB. Prognostic factors in pediatric brain-stem gliomas. J Neurosurg 1986;65:751-5. 
9. Fisher PG, Breiter SN, Carson BS, et al. A clinicopathologic reappraisal of brain stem tumor classification. Identification of pilocystic astrocytoma and fibrillary astrocytoma as distinct entities. Cancer 2000;89:1569-76.

10. Gururangan S, McLaughlin CA, Brashears J, et al. Incidence and patterns of neuraxis metastases in children with diffuse pontine glioma. J Neurooncol 2006;77:207-12.

11. Fischbein NJ, Prados MD, Wara W, Russo C, Edwards MS, Barkovich AJ. Radiologic classification of brain stem tumors: correlation of magnetic resonance imaging appearance with clinical outcome. Pediatr Neurosurg 1996;24:9-23.

12. Albright AL, Packer RJ, Zimmerman R, Rorke LB, Boyett J, Hammond GD. Magnetic resonance scans should replace biopsies for the diagnosis of diffuse brain stem gliomas: a report from the Children's Cancer Group. Neurosurgery 1993;33:1026-9; discussion 1029-30.

13. Roujeau T, Machado G, Garnett MR, et al. Stereotactic biopsy of diffuse pontine lesions in children. J Neurosurg 2007;107(Suppl 1):1-4.

14. Geoerger B, Hargrave D, Thomas F, et al.; ITCC (Innovative Therapies for Children with Cancer) European Consortium. Innovative Therapies for Children with Cancer pediatric phase I study of erlotinib in brainstem glioma and relapsing/refractory brain tumors. Neuro Oncol 2011;13:109-18.

15. MacDonald TJ. Diffuse intrinsic pontine glioma (DIPG): time to biopsy again? Pediatr Blood Cancer 2012;58:487-8.

16. Janssens GO, Jansen MH, Lauwers SJ, et al. Hypofractionation vs conventional radiation therapy for newly diagnosed diffuse intrinsic pontine glioma: a matched-cohort analysis. Int J Radiat Oncol Biol Phys 2013;85:315-20.

17. Jansen MH, van Vuurden DG, Vandertop WP, Kaspers GJ. Diffuse intrinsic pontine gliomas: a systematic update on clinical trials and biology. Cancer Treat Rev 2012;38:27-35.

18. Hall WA, Doolittle ND, Daman M, et al. Osmotic blood-brain barrier disruption chemotherapy for diffuse pontine gliomas. J Neurooncol 2006;77:279-84.

19. Warren K, Jakacki R, Widemann B, et al. Phase II trial of intravenous lobradimil and carboplatin in childhood brain tumors: a report from the Children’s Oncology Group. Cancer Chemother Pharmacol 2006;58:343-7.

20. Greenberg ML, Fisher PG, Freeman C, et al. Etoposide, vincristine, and cyclosporin A with standard-dose radiation therapy in newly diagnosed diffuse intrinsic brainstem gliomas: a pediatric oncology group phase I study. Pediatr Blood Cancer 2005;45:644-8.

21. Bobo RH, Laske DW, Akbasak A, Morrison PF, Dedrick RL, Oldfield EH. Convection-enhanced delivery of macromolecules in the brain. Proc Natl Acad Sci USA 1994;91:2076-80.

22. Anderson RC, Kennedy B, Yanes CL, et al. Convection-enhanced delivery of topotecan into diffuse intrinsic brainstem tumors in children. J Neurosurg Pediatr 2013;11:289-95.

23. Paugh BS, Broniscer A, Qu C, et al. Genome-wide analyses identify recurrent amplifications of receptor tyrosine kinases and cell-cycle regulatory genes in diffuse intrinsic pontine glioma. J Clin Oncol 2011;29:3999-4006.

24. Zarghooni M, Bartels U, Lee E, et al. Whole-genome profiling of pediatric diffuse intrinsic pontine gliomas highlights platelet-derived growth factor receptor alpha and poly (ADP-ribose) polymerase as potential therapeutic targets. J Clin Oncol 2010;28:1337-44.

25. Puget S, Philippe C, Bax DA, et al. Mesenchymal transition and PDGFRA amplification/mutation are key distinct oncogenic events in pediatric diffuse intrinsic pontine gliomas. PLoS ONE 2012;7:e30313.

26. Verhaak RG, Hoadley KA, Purdom E, et al.; Cancer Genome Atlas Research Network. Integrated genomic analysis identifies clinically relevant subtypes of glioblastoma characterized by abnormalities in PDGFRA, IDH1, EGFR, and NF1. Cancer Cell 2010;17:98-110.

27. Grill J, Puget S, Andreiuolo F, Philippe C, MacConaill L, Kieran MW. Critical oncogenic mutations in newly diagnosed pediatric diffuse intrinsic pontine glioma. Pediatr Blood Cancer 2012;58:489-91.

28. Khuong-Quang DA, Buczkowicz P, Rakopoulos P, et al. K27M mutation in histone H3.3 defines clinically and biologically distinct subgroups of pediatric diffuse intrinsic pontine gliomas. Acta Neuropathol 2012;124:439-47.

29. Warren KE, Killian K, Suuriniemi M, Wang Y, Quezado M, Meltzer PS. Genomic aberrations in pediatric diffuse intrinsic pontine gliomas. Neuro Oncol 2012;14:326-32.
30. Wu G, Broniscer A, McEachron TA, et al.; St. Jude Children's Research Hospital-Washington University Pediatric Cancer Genome Project. Somatic histone $\mathrm{H} 3$ alterations in pediatric diffuse intrinsic pontine gliomas and non-brainstem glioblastomas. Nat Genet 2012;44:251-3.

31. Barski A, Cuddapah S, Cui K, et al. High-resolution profiling of histone methylations in the human genome. Cell 2007;129:823-37.

32. Reynolds N, Salmon-Divon M, Dvinge H, et al. NuRD-mediated deacetylation of H3K27 facilitates recruitment of Polycomb Repressive Complex 2 to direct gene repression. EMBO J 2012;31:593-605.

33. Lewis PW, Müller MM, Koletsky MS, et al. Inhibition of PRC2 activity by a gain-of-function $\mathrm{H} 3$ mutation found in pediatric glioblastoma. Science 2013;340:857-61.

34. Monje M, Mitra SS, Freret ME, et al. Hedgehog-responsive candidate cell of origin for diffuse intrinsic pontine glioma. Proc Natl Acad Sci USA 2011;108:4453-8.

35. Becher OJ, Hambardzumyan D, Walker TR, et al. Preclinical evaluation of radiation and perifosine in a genetically and histologically accurate model of brainstem glioma. Cancer Res 2010;70:2548-57.

36. Schwartzentruber J, Korshunov A, Liu XY, et al. Driver mutations in histone H3.3 and chromatin remodelling genes in paediatric glioblastoma. Nature 2012;482:226-31.

37. Paugh BS, Qu C, Jones C, et al. Integrated molecular genetic profiling of pediatric high-grade gliomas reveals key differences with the adult disease. J Clin Oncol 2010;28:3061-8.

38. Raffel C, Frederick L, O'Fallon JR, et al. Analysis of oncogene and tumor suppressor gene alterations in pediatric malignant astrocytomas reveals reduced survival for patients with PTEN mutations. Clin Cancer Res 1999;5:4085-90.

39. Barrow J, Adamowicz-Brice M, Cartmill M, et al. Homozygous loss of ADAM3A revealed by genome-wide analysis of pediatric high-grade glioma and diffuse intrinsic pontine gliomas. Neuro Oncol 2011;13:212-22.

40. Arjona D, Bello MJ, Alonso ME, et al. Molecular analysis of the EGFR gene in astrocytic gliomas: mRNA expression, quantitative-PCR analysis of non-homogeneous gene amplification and DNA sequence alterations. Neuropathol Appl Neurobiol 2005;31:384-94.

41. Pollack IF, Hamilton RL, James CD, et al.; Children's Oncology Group. Rarity of PTEN deletions and EGFR amplification in malignant gliomas of childhood: results from the Children's Cancer Group 945 cohort. J Neurosurg 2006;105(Suppl 5):418-24.

42. Louis DN, Rubio MP, Correa KM, Gusella JF, von Deimling A. Molecular genetics of pediatric brain stem gliomas. Application of PCR techniques to small and archival brain tumor specimens. J Neuropathol Exp Neurol 1993;52:507-15

43. Wong KK, Tsang YT, Chang YM, et al. Genome-wide allelic imbalance analysis of pediatric gliomas by single nucleotide polymorphic allele array. Cancer Res 2006;66:11172-8.

44. Gilbertson RJ, Hill DA, Hernan R, et al. ERBB1 is amplified and overexpressed in high-grade diffusely infiltrative pediatric brain stem glioma. Clin Cancer Res 2003;9(10 Pt 1):3620-4.

45. Pollack IF, Hamilton RL, Sobol RW, et al.; Children's Oncology Group. IDH1 mutations are common in malignant gliomas arising in adolescents: a report from the Children's Oncology Group. Childs Nerv Syst 2011;27:87-94.

46. Yan H, Parsons DW, Jin G, et al. IDH1 and IDH2 mutations in gliomas. N Engl J Med 2009;360:765-73.

47. Pollack IF, Finkelstein SD, Burnham J, et al.; Children's Cancer Group. Age and TP53 mutation frequency in childhood malignant gliomas: results in a multi-institutional cohort. Cancer Res 2001;61:7404-7.

48. Milinkovic V, Bankovic J, Rakic M, et al. Genomic instability and p53 alterations in patients with malignant glioma. Exp Mol Pathol 2012;93:200-6.

49. Fontebasso AM, Schwartzentruber J, Khuong-Quang DA, et al. Mutations in SETD2 and genes affecting histone H3K36 methylation target hemispheric high-grade gliomas. Acta Neuropathol 2013;125:659-69.

50. Rickert $\mathrm{CH}$, Sträter R, Kaatsch P, et al. Pediatric high-grade astrocytomas show chromosomal imbalances distinct from adult cases. Am J Pathol 2001;158:1525-32. 